\title{
Oceanic Flights and Airspace: Improving Efficiency by Trajectory-Based Operations
}

\author{
Alicia Borgman Fernandes ${ }^{1}$ and Juan Rebollo ${ }^{2}$ \\ Mosaic ATM, Leesburg, VA, 20175 \\ and \\ Michael Koch ${ }^{3}$ \\ NASA Langley Research Center, Hampton, VA, 23681
}

\begin{abstract}
Oceanic operations suffer from multiple inefficiencies, including pre-departure planning that does not adequately consider uncertainty in the proposed trajectory, restrictions on the routes that a flight operator can choose for an oceanic crossing, time-consuming processes and procedures for amending en route trajectories, and difficulties exchanging data between Flight Information Regions (FIRs). These inefficiencies cause aircraft to fly suboptimal trajectories, burning fuel and time that could be conserved. A concept to support integration of existing and emerging capabilities and concepts is needed to transition to an airspace system that employs Trajectory Based Operations (TBO) to improve efficiency and safety in oceanic operations. This paper describes such a concept and the results of preliminary activities to evaluate the concept, including a stakeholder feedback activity, user needs analysis, and high level benefits analysis.
\end{abstract}

\section{Nomenclature}

$\begin{array}{ll}\text { 4DT } & =\text { 4-Dimensional Trajectory } \\ \text { ASP } & =\text { ATM Service Provider } \\ \text { ATM } & =\text { Air Traffic Management } \\ \text { ConOps } & =\text { Concept of Operations } \\ \text { DARP } & =\text { Dynamic Airborne Reroute Procedure } \\ \text { FF-ICE } & =\text { Flight and Flow }- \text { Information for a Collaborative Environment } \\ \text { FIR } & =\text { Flight Information Region } \\ \text { FOC } & =\text { Flight Operations Center (also Airline Operational Control, AOC) } \\ \text { ICAO } & =\text { International Civil Aviation Organization } \\ \text { JPDO } & =\text { Joint Planning and Development Office } \\ \text { SWIM } & =\text { System Wide Information Management } \\ \text { TBO } & =\text { Trajectory Based Operations } \\ \text { UPR } & =\text { User Preferred Route }\end{array}$

\section{Introduction}

$\mathrm{O}$ CEANIC operations suffer from multiple inefficiencies, including pre-departure planning that does not adequately consider uncertainty in the proposed trajectory, restrictions on the routes that flight operators can choose for oceanic crossings, time-consuming processes and procedures for amending en route trajectories, and difficulties exchanging data between Flight Information Regions (FIRs). These inefficiencies cause aircraft to fly suboptimal trajectories, burning fuel and time that could be conserved. There are emerging capabilities that can be leveraged to support improvements in many of these areas. To date, initiatives to leverage these capabilities tend to focus on implementation of one technology to improve efficiency in one dimension, possibly even in only one region. There have been few, if any, initiatives to apply strategic planning principles to Trajectory Based Operations (TBO)

\footnotetext{
${ }^{1}$ Principal Analyst, 540 Fort Evans Road NE Ste 300, AIAA Member.

${ }^{2}$ Senior Analyst, 540 Fort Evans Road NE Ste 300.

${ }^{3}$ Aerospace Engineer, Crew Systems and Aviation Operations Branch, Mail Stop 152, AIAA Member.
}

American Institute of Aeronautics and Astronautics 
in oceanic airspace or to support collaborative flight planning across international boundaries. This paper presents a concept for applying TBO to oceanic operations in the mid-term time frame. The concept incorporates multiple emerging concepts and capabilities into a single concept and applies to Pacific and Atlantic operations.

\section{Background}

One goal of the Next Generation Air Transportation System (NextGen) is to allow aircraft to fly trajectories as close as possible to flight operator-preferred trajectories. ${ }^{1}$ Consistent with the International Civil Aviation Organization's (ICAO's) vision for global Air Traffic Management (ATM), ${ }^{2}$ this is accomplished through the transition to Trajectory Based Operations (TBO) and the use of 4-D trajectories (4DTs). This section provides brief introductions to 4D trajectories and TBO as described by FAA and ICAO documentation. It also provides some background on oceanic operations, particularly those aspects that differ from domestic operations.

\section{A. 4-D Trajectories and Trajectory Based Operations}

The NextGen Concept of Operations (ConOps) defines the 4DT as "a precise description of an aircraft path in time and space: the 'centerline' of a path plus the position uncertainty, using waypoints to describe specific steps along the path... containing altitude descriptions and the time(s) the trajectory will be executed. The required level of specificity of the 4DT depends on the flight operating environment." 3 This definition focuses on the increased predictability provided by a 4DT and the ability to use this improved predictability to increase capacity and trajectory efficiency. Figure 1 illustrates the Joint Planning and Development Office (JPDO) vision for the 4DT. Note the uncertainty around the top of descent point for the

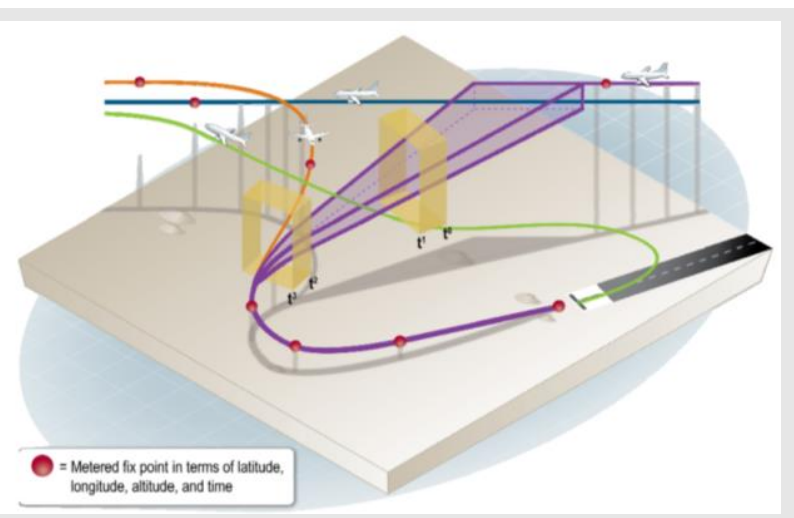

Figure 1: 4DT as illustrated in the NextGen ConOps ${ }^{3}$ aircraft preparing for arrival (trajectory is represented in purple) and greater precision for all other aspects of the trajectory. Also note the rectangular bounds around that trajectory for separation management.

In the early planning stages, a flight's 4DT is subject to uncertainty from a variety of sources, including weather, aircraft weight and balance, aircraft readiness, and air traffic. However, proposed flight plans are evaluated deterministically; current automation does not consider trajectory-specific uncertainty. Instead, trajectory uncertainty (and the potential for loss of communications) is taken into account through the use of large separation minima (ranging from 30-80 $\mathrm{nm}$ in most oceanic regions) and procedural control, in which flight crews are expected to maintain the assigned speed (Mach number) and altitude, and report their position at specified intervals. ${ }^{4}$ Mitchell and Ohsfeldt ${ }^{5}$ described one approach to explicitly incorporate 4DT uncertainty in developing a strategic ATM plan for oceanic airspace composed of a set of 4DTs close to one of several trajectory alternatives proposed by the flight operator. Uncertainty was expressed as lateral and time windows about a given waypoint that the flight operator considered acceptable. Rebollo and Brinton ${ }^{6}$ described a different approach in which demand and capacity for a given airspace resource were determined probabilistically for the purpose of developing, evaluating, and amending ATM plans. The Oceanic TBO concept incorporates a method like that of Rebollo and Brinton, in which strategic oceanic ATM planning uses demand and capacity forecasts to identify imbalances and introduce controls where necessary to adjust demand to reduce the risks of both over- and under-delivery to the airspace in question.

As time progresses, TBO expects that the 4DT will be dynamically adjusted as needed to account for new information, although it is not clear that this includes reducing the volume representing the position uncertainty as aircraft performance parameters become known. If so, it is expected that this will be done via negotiation. The Oceanic TBO concept also calls for flight operators to provide updates to the 4DT, particularly trajectory uncertainty, as more information about airspace conditions and other factors affecting aircraft performance become known. Similarly, ASPs are expected to provide updated information about constraints affecting the agreed 4DT, consistent with ICAO guidelines. ${ }^{7}$ However, current processes for negotiating flight plan amendments are time-consuming and can be workload-intensive, and operational personnel have little confidence in the idea that the trajectory will be easily updated - especially after departure. Thus, the Oceanic TBO concept proposes procedures for explicitly updating aspects of the 4DT that are subject to the greatest uncertainty, particularly altitude, based on events that dramatically reduce uncertainty (e.g., top of climb). This is especially important given the duration of most transoceanic flights and the particulars of procedures for traversing oceanic airspace.

American Institute of Aeronautics and Astronautics 


\section{B. Oceanic Operations}

In today's environment, oceanic operations are characterized by procedural control associated with historical deficiencies in surveillance and communication capabilities and resulting in large separation minima. Track systems and international boundary crossings constrain flight operators' route choices. Further, route amendments require international coordination across systems with varying capabilities.

\section{Oceanic Track Systems}

In many regions of the Pacific and Atlantic, traffic is organized along tracks that reduce the opportunity for trajectory conflicts in procedurally controlled airspace. Aircraft are assigned a track prior to entering the airspace, along with a speed to maintain in order to maintain longitudinal separation. Once the aircraft enters the tracks it may be unable to climb to a more desirable altitude due to traffic. The goal is to manage high volume while allowing aircraft to use efficient routes. Organizing traffic in a track system manages complexity for controllers but constrains the ability for flight operators to use preferred (typically the most fuel-efficient) routes.

User Preferred Routes (UPRs) are supported in much of the Pacific and outside the track system in New York Oceanic airspace, with efforts underway to increase support for them. However, UPRs are typically supported for only a portion of a flight's route, requiring the flight operator to align the route to transition onto and/or off of an organized track. Aircraft operating on an inner track of a set of organized tracks - typically the most efficient airspace - are unable to exit the tracks to exercise UPR, although they may be able to transition to an adjacent track. In addition, in many cases there are limitations on oceanic boundary crossing points between FIRs. Even in regions where the airspace can support UPRs, many aircraft and/or flight operator systems may be unable to do so. ${ }^{8}$

\section{Inter-FIR Coordination}

Limitations in inter-FIR coordination, including between oceanic and domestic areas within the same country, coupled with limitations in traffic management planning capabilities, mean that many aircraft are unable to receive their full route in the departure clearance. Typically, the aircraft receives its departure clearance from the departure airport to the oceanic entry point unless the departure airport is within a certain distance from the oceanic entry point. The flight crew requests the oceanic clearance when en route to the oceanic entry point. The oceanic clearance provides a specific flight level and cruise speed, which may not match the oceanic entry point or the flight level included in the flight plan.

The oceanic clearance may include a gateway reservation - a 10-minute window during which the flight is expected to arrive at the oceanic entry point in order to ensure 10 minutes longitudinal separation at the first fix on the oceanic track. ${ }^{9}$ However, flight operators often fail to meet these times for a number of reasons including incorrectly predicted push back time and poor predictions of delays and route changes that will be encountered on the surface and en route to the oceanic gateway. When a flight operator determines that it will miss a gateway reservation, it must cancel the current reservation and request an entirely new gateway reservation. There currently is no other process for updating the time at which the aircraft will enter oceanic airspace. The new reservation often involves a suboptimal track assignment or holding at the track entry point.

An additional effect of poor inter-FIR coordination is that traffic planning systems may not receive updated information about aircraft en route to US managed oceanic airspace, particularly aircraft that will transit US oceanic airspace without landing at a US airport. This constrains the ability to plan traffic flows, particularly when constraints such as weather require flights to deviate.

Inadequate inter-FIR coordination also is a significant barrier to trajectory modification. Oceanic reroutes often traverse multiple FIRs. En route trajectory amendments in oceanic airspace are governed by the Dynamic Airborne Reroute Procedure (DARP). ${ }^{10}$ While DARP allows en route trajectory amendments, the procedure is time-consuming, requiring the dispatcher to coordinate with the FIR where the aircraft is currently operating and all downstream FIRs, possibly by e-mail, before the light crew can request the route amendment. New York and Oakland Oceanic have developed procedures for coordinating with their international counterparts for some common routes and continue to work to improve the implementation of the procedure, but the significant amount of coordination required makes the procedure time consuming.

\section{En Route Trajectory Amendments}

Difficulty in coordinating en route trajectory amendments is further compounded by flight operator planning capabilities designed to identify routes from the departure airport to the destination airport. They are not typically well suited to identifying optimal routes from an aircraft's current location. This limits flight operators' ability to amend trajectories while aircraft are en route, and causes missed opportunities for moving long-haul aircraft to more efficient trajectories when new information (e.g., updated winds) becomes available. Even if FOC tools support dynamic reroutes, dispatcher workload often prevents them from generating and managing DARP requests. ${ }^{11}$ In addition, ATC

infrastructure is often unable to review and approve amendments in a timely manner upon aircrew request. These are disincentives for flight operators to invest in infrastructure to support dynamic reroutes.

American Institute of Aeronautics and Astronautics 
Recently, NASA has pursued a concept to support dispatcher identification of an optimized weather reroute from the aircraft's current position to a downstream fix. ${ }^{12,13}$ Coupling this with a different NASA concept for aircraft automation to identify an optimal route from the aircraft's current location to the desired downstream location, ${ }^{14}$ and the desired 4DT amendment can be readily identified. The Oceanic TBO concept, then, must address difficulties associated with the flight operator coordinating the 4DT amendment with the ASP.

\section{Oceanic TBO Concept}

The Oceanic TBO concept describes how TBO can be applied to the oceanic environment to better accommodate flight operator preferences and improve trajectory management. It incorporates various existing NASA and FAA research concepts into a single, coherent concept. In this section, we summarize key aspects of the Oceanic TBO concept that stand out from previous definitions of TBO.

\section{A. Strategic Planning using 4-D Trajectories}

The vision of 4DTs is one of decreased uncertainty in the aircraft trajectory due to advancements such as improved precision in aircraft navigation performance, more frequent and precise position data, and the ability to share trajectory information in near real time. However, the oceanic portion of a transoceanic trajectory may be planned more than 12 hours before the aircraft enters the airspace of interest. In this timeframe, the 4DT may contain significant uncertainty due to multiple sources unrelated to aircraft navigation performance. In particular, the location at which a climb in oceanic cruise should be initiated to achieve the optimal vertical profile depends greatly on factors that may not be known until shortly before departure (e.g., final weight and balance) or even after the aircraft is in the oceanic airspace (e.g., precise wind information and turbulence reports).

Oceanic TBO proposes strategic flow planning that explicitly considers 4DT uncertainty beyond that of the aircraft's navigation performance. The ATM system will use a collection of proposed 4DTs and characteristics of 4DT uncertainty to derive a probabilistic demand forecast supporting identification of demand/capacity imbalances. Initiatives can reduce expected demand relative to expected capacity in a way that explicitly considers uncertainty as well as the ability for the system to apply some delay tactically to avoid over-constraining traffic flows. Such tactical delays can take the form of en route metering or vectoring and/or speed controls like those routinely applied by controllers today. However, the tactical delay expected to be absorbed is limited to manage controller complexity. Allowing the expected demand to exceed capacity at a critical resource by a small amount can prevent loss of available capacity without overwhelming controllers with aircraft that must tactically absorb delay. ${ }^{6}$

As time progresses, uncertainty will be reduced in two ways. First, the ATM system's probabilistic demand prediction will use historical data to narrow uncertainty based on lead time and events associated with preparing a flight for departure. In the TBO sense, the "tube" surrounding the cleared 4DT is automatically shrunk to reflect the reduced uncertainty and the probabilistic airspace demand forecast is updated accordingly.

Second, flight operators will propose updates to the 4DT to reflect flight progress. For example, once the final weight and balance is known, the flight operator will have greater confidence in the optimal oceanic flight profile and can propose an updated 4DT reflecting the new information. The flight operator can submit another update when the aircraft reaches top of climb, which still may be several hours before the aircraft reaches oceanic airspace. Such updates are an expected part of TBO and therefore must be able to be proposed, de-conflicted, negotiated, approved, and clearances delivered to the flight deck quickly and efficiently. Note that 4DTs must be de-conflicted over some planning horizon that, for most of a long-haul flight, does not represent the full 4DT to the destination.

\section{B. Expanded Use of User Preferred Routes}

Full implementation of TBO in oceanic airspace will allow UPRs to be the norm throughout US-managed airspace in the Pacific and Atlantic regions. For most flights the user-preferred route will represent the fuel optimal route, although flight operators also have considerations such as overflight fees and maintaining network schedule integrity. Where necessary, 4DTs will be restricted to ensure safety and organize traffic flows, but these restrictions will be minimal. Restrictions will chiefly support merging and spacing of traffic transitioning between oceanic and domestic airspace, particularly for arrival at (and departure from) airports located close to the boundary between domestic and oceanic airspace. Note that this will require improved coordination with neighboring FIRs to relax restrictions on boundary crossings, as well as improved 4DT management and support for UPRs in other FIRs.

Previous researchers have estimated the improved efficiency associated with allowing aircraft to fly wind-optimal routes in oceanic airspace, ${ }^{15,16}$ and several demonstrations have shown that significant efficiency can be realized by allowing aircraft to fly UPRs. ${ }^{17,18,19}$ However, these studies have done little to explore the effect of widespread use of UPRs, although this has been a topic of discussion at industry meetings. ${ }^{20,21}$

American Institute of Aeronautics and Astronautics 
A potential side effect of widespread UPR use is increased congestion. One Stakeholder Feedback Activity participant said, "Flight planning engines put all the aircraft in the same place." UPRs may involve 4DTs that intersect at multiple locations, altitudes, and angles. This would increase complexity of traffic flows for controllers, potentially meaning that "no one will get their preferred route because of the conflicts," according to another Stakeholder. Increasing traffic complexity may decrease the number of aircraft a single controller can manage (when traffic is organized on oceanic tracks, a single controller may manage up to 70 aircraft). This could increase staffing cost, limit capacity gains from reduced separation minima, and/or result in airborne reroutes, holding, and other side effects of overloaded sectors. Some technological improvements could mitigate these side effects. For example, increasing the resolution of turn point definitions used by flight planning engines - and ensuring that the ATM system can process 4DTs proposed with increased resolution - could separate some proposed 4DTs from each other.

\section{Trajectory Negotiation and Dynamic Trajectory Management}

Trajectory negotiation is fundamental to TBO. Negotiation will start when the flight operator submits a preliminary flight plan and will continue throughout the flight. To agree upon a transoceanic 4DT, all FIRs that the aircraft will traverse must approve the portion of the 4DT traversing their airspace. ICAO provisions do not require ASPs to coordinate the suitability of a 4DT with each other, but do allow for an ASP to provide such a service. ${ }^{7}$

Consistent with FF-ICE, ${ }^{7}$ the Oceanic TBO concept expects that ASPs will publish flight planning constraints via SWIM, allowing flight operators to access all known constraints during flight planning and trajectory negotiation. FAA will publish all known constraints affecting US-managed airspace, and the SWIM infrastructure will support flight operators (and FAA) receiving constraint information from other SWIM-compliant ASPs. Flight operators will incorporate these constraints into their planning and identify the best available 4DT for each flight. Flight operators will submit the proposed 4DT to the ASP where the departure airport is located, and the ASP's SWIM implementation will publish the proposed 4DT so that all ASPs whose airspace will be affected by the flight plan trajectory can review and accept or reject it. This is a departure from current operations, in which the dispatcher files a flight plan with the departure ASP, which typically forwards the flight plan to the next FIR to be traversed a pre-coordinated amount of time (e.g., 60 minutes) before the flight enters the neighboring FIR. This simplifies the flight plan filing process for flight operators but does not provide strategic planning data to downstream FIRs and limits their ability to provide flight operators information about constraints affecting the flight plan.

If the flight plan is unacceptable to one or more ASPs, the ASP(s) in question will describe the violated constraint(s) and may propose an alternate 4DT. It is expected that negotiation is mostly automated, with the flight operator's planning system automatically adjusting the proposed 4DT and submitting a new proposal for evaluation by all affected ASPs. The ATM system, in turn, automatically evaluates the proposed 4DT and either approves it or replies with the constraints affecting it. Each flight operator and ASP may set its own expectations for human involvement in negotiation. The end result is that the flight plan will comprise a gate-to-gate 4DT coordinated among all affected ASPs and the flight operator that will meet all known airspace and surface constraints. If one or more ASP does not participate in pre-departure data exchange and coordination, a SWIM service can forward the filed flight plan to the appropriate address so that the flight operator only will have to file the flight plan once.

Figure 2 shows a notional approach to providing visibility into 4DT negotiation. ASPs evaluate a proposed 4DT and accept it or add constraints. The capability supports FAA as well as ICAO collaborative flight planning and data exchange goals and will decrease the time and workload required to coordinate 4 DTs and amendments.

When an ASP adds a constraint that the 4DT must satisfy, the result may require an amendment to the 4DT in a different FIR as well. Multi-party trajectory negotiation via SWIM will support coordinating such amendments and result in more efficient 4DTs. Such a 4DT negotiation capability must allow each stakeholder to perform actions such as:

- View proposed 4DT.

- Evaluate 4DT against desired criteria.

- Provide an indication of conflicts with other 4DTs and with airspace constraints.

- Add constraints the proposed 4DT must satisfy (e.g., altitude restriction to resolve a conflict).

- Propose further amendments to the 4DT.

- Approve the 4DT.

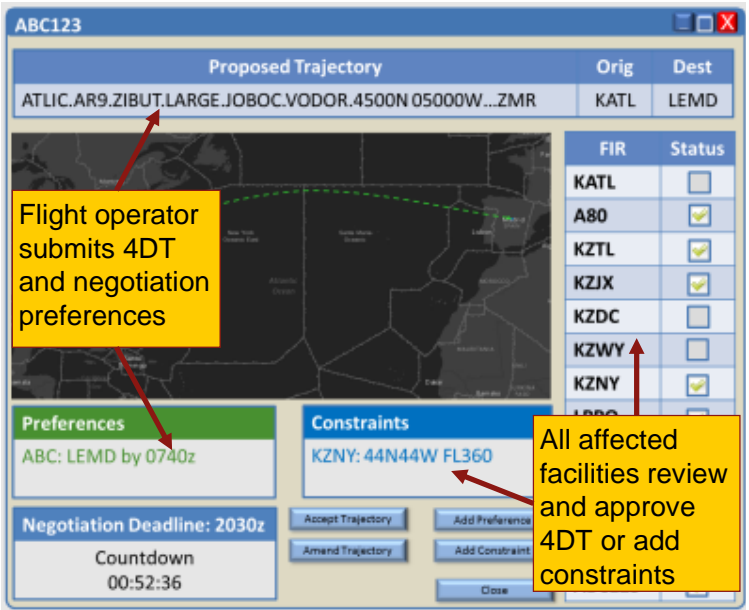

Figure 2: Multi-party trajectory negotiation 
The negotiation capability will track which stakeholders must approve each 4DT, monitor the time available for negotiation, and manage notifications to facilitate stakeholder participation in negotiation. Advanced automation for 4DT prediction, evaluation, and generation can support negotiation while minimizing workload. Additional research will be required to determine the best approach to selecting a 4DT if multiple ASPs are affected and no agreement is reached in the time available. One possible approach is to prioritize the violated constraints and resolve them in a piecewise fashion. This will likely require further coordination and negotiation later in the flight, but if the tradeoff is between safety of flight and efficiency, safety must take priority. FAA could implement such a capability as an extension of its FF-ICE compliant planning and filing service, or flight operators could implement a capability that subscribes to constraints and the 4DT planning and filing status published by ASPs.

\section{Trajectory Conformance Monitoring}

In current operations, flight crews are rarely provided clearances that include multiple flight level changes. However, the 4DT by definition includes the flight's altitude profile. Incorporating the altitude profile into the clearance provided to the flight crew will require a fundamental shift in the way air traffic control is performed and strategies and tools controllers currently use for conflict detection and resolution.

Aircraft already precisely follow lateral trajectories, meet timing constraints, and automatically execute direction and speed changes. However, they do not automatically execute flight level changes in the cruise phase and there are no standards in development for them to do so in the future. Previous TBO efforts ${ }^{22}$ discuss vertical conformance requirements, but ignore the operational question of when the flight should receive clearance to execute a flight level change. Thus, 4DT conformance will require careful consideration of the $4^{\text {th }}$ dimension - in this case, altitude. The definition of TBO requires use of the altitude profile for planning and conflict detection. Thus, if the aircraft deviates from the altitude profile, it is out of conformance with the 4DT. On the other hand, currently flight crews and ATC perform just-in-time coordination of flight level changes to ensure consistency (there have been issues with flight crews incorrectly executing flight level change clearances, leading to safety risks).

A balance needs to be struck such that the flight operator can provide its optimal altitude profile to the ASP so that the ASP can use it for planning traffic flows, negotiating individual 4DTs, and preventing conflicts. However, TBO procedures may need to support just-in-time flight level change negotiations, including those involving the use of block altitudes. Participants in the Stakeholder Feedback Activity were comfortable with an approach by which the aircraft automation would remind the flight crew of the agreed flight level change and the flight crew would downlink their intent to execute the maneuver some time or distance - e.g., 10 minutes - before beginning the maneuver. This will help to prevent flight crew errors of omission and validate the trajectory synchronization information in the ground automation. Such procedures also would help ensure that the flight operator negotiate a trajectory amendment if the altitude profile needs to be modified for the trajectory to remain desirable.

\section{Requirements to Support Participation in Oceanic TBO}

The research team performed several activities as part of concept development and initial concept evaluation, including identification of current shortfalls, elicitation of stakeholder feedback, analysis of user needs, and preliminary cost and benefits analyses. Each of these analyses built on each other. For brevity, these activities are discussed here in terms of requirements to support operational participation in Oceanic TBO and expected benefits mechanisms. These requirements were developed as a result of the User Needs Analysis, which built on the shortfall analysis as well as the concept and results from the Stakeholder Feedback Activity. (The Stakeholder Feedback Activity elicited feedback on the shortfalls and the concept from pilots, dispatchers, FAA oceanic traffic management personnel, and an International Air Transport Association representative to the ATCSCC.)

The analysis focused on 4 different classes of users: air traffic controllers, traffic management personnel, flight crews, and flight dispatchers. User needs were defined as shortfalls in the current operation (i.e., a currently unmet need) and requirements to support the improvements envisioned by the Oceanic TBO concept (information requirements, coordination requirements, and automation support requirements). The research team walked through scenarios used for concept development and the Stakeholder Feedback Activity and asked questions such as:

- What task or goal does the user(s) need to accomplish?

- What information does the user(s) need?

- With whom does the user(s) need to coordinate/collaborate?

- What automation support does the user(s) need?

Requirements were classified as to whether they supported planning tasks of trajectory management or negotiation tasks. Automation requirements were further classified according to data exchange automation and decision support automation. Results are summarized here for each category of user.

American Institute of Aeronautics and Astronautics 


\section{A. Traffic Managers}

As part of their strategic planning responsibility, traffic managers are expected to coordinate constraints, ATM plans, 4DTs, and 4DT amendments with upstream and downstream FIRs as well as flight operators. All of these may have different automation capabilities. Traffic managers also are expected to publish constraints and ATM plans as soon as they are known. To do this, they will need demand and capacity information, including uncertainty, for flights planning to traverse the airspace. They will also need information about all constraints affecting 4DTs in the airspace of interest, including constraints affecting upstream and downstream FIRs and 4DT amendments from other FIRs that affect the 4DT in the airspace of interest. Traffic managers will need automation support for exchanging constraint and 4DT data with flight operators (FOC and flight crew) and neighboring FIRs, negotiating 4DTs with flight operators, and will require automation that can:

- Retain and process full 4DTs

- Consider 4DT uncertainty in modeling and balancing demand and capacity

- Support evaluation of the effects of constraints and ATM actions on 4DTs

- Synchronize air and ground 4DTs

- "Tune" 4DTs as more information becomes known and uncertainty decreases

- Amend the portion of the 4DT traversing the local airspace while the aircraft is in an upstream FIR

\section{B. Air Traffic Controllers}

Air traffic controllers will be expected to support 4DT negotiation with flight crews, including for amendments that affect the 4DT in downstream sectors and/or FIRs. They also are expected to provide a safe and legal weather avoidance clearance in all cases and accept flights crossing sector and/or FIR boundaries at flight operator preferred locations, rather than only pre-coordinated gateways. To support these coordination tasks, controllers will need information about constraints and their effects on 4DTs, suitability of proposed 4DT amendments, when (and why) aircraft will be unable to conform to the cleared 4DT, and flight crew requirements for severe weather avoidance. They will need automation that supports easy evaluation of proposed 4DT amendments across sector and/or FIR boundaries, exchanging complex 4DTs with aircraft, and exchanging 4DTs and constraints with international partners. The automation also must support air/ground trajectory synchronization.

\section{Dispatchers}

Dispatchers will need to ensure that 4DTs and flight operator preferences are provided to all affected ASPs predeparture and be able to efficiently negotiate 4DT amendments with all affected ASPs (and flight crew) during any phase of flight. To do this, they will need information about weather and ATM constraints affecting flights' optimal 4DTs across all FIRs the 4DTs traverse, as well as expected ATM strategies to manage those constraints and their effects on individual 4DTs and the flight operator network. They also need automation supporting full 4DT planning, including uncertainty, and probing for and resolving conflicts across sector and FIR boundaries to evaluate 4DT amendments. Dispatchers will need to be able to exchange complex 4DTs with aircraft as well as exchange trajectory and constraint information (and expected effects on 4DTs) with all ASPs the aircraft will traverse.

\section{Flight Crews}

Under the Oceanic TBO concept, flight crews will require improved support for 4DT planning, negotiation, and conformance. They need information about the suitability of the current and proposed 4DTs given airspace conditions and aircraft capabilities. This will require flight crews to receive updated weather forecasts, pilot reports, and other information about downstream airspace conditions, even when they are operating in remote airspace. To support en route reroutes, flight crews need automation that can generate optimized 4DTs from the aircraft's current location that meet weather and ATM constraints and flight operator preferences (e.g., Ref. 14). (Although the dispatcher retains joint responsibility for safety of flight and performs most of the pre-flight 4DT planning, at times it is beneficial for the flight crew to engage in reroute planning and therefore the Oceanic TBO concept expects them to have the tools to do so.) The flight crew needs to be able to receive complex 4DTs and auto-load them into the FMS for evaluation, including comparing them with the current flight plan and aircraft performance. They need to be able to downlink a proposed 4DT as a request to ATC, even if it is a complex 4DT. When they downlink the request, they need a quick response from ATC, particularly when requesting a weather deviation. When operating in airspace affected by weather, they need to be able to communicate to ATC their requirements for weather deviation. After negotiation is complete, they need to be able to efficiently execute the amendment. While flying a 4DT, the flight crew needs procedures for executing altitude changes and other maneuvers to maintain conformance with the 4DT, and to be notified as soon as the aircraft detects that it will be unable to conform to the 4DT in order to make corrections and

American Institute of Aeronautics and Astronautics 
coordinate with ATC as needed. When the aircraft cannot conform to the 4DT and the flight crew is unable to coordinate an amendment with ATC, the flight crew will need procedures to maintain safety of flight.

\section{Expected Oceanic TBO Benefits}

The Oceanic TBO concept is expected to provide a range of benefits. These expectations were shared with stakeholders in our feedback sessions, and the input received, along with the results of the user needs analysis, allowed the research team to trace paths from the concept through a set of benefits mechanisms to associated benefits and performance metrics that are considered useful for the full benefits assessment task. In addition, each benefits mechanism was assessed qualitatively, and available associated quantitative information was also provided. (A thorough quantitative benefits analysis was beyond the scope of the project.) Quantitative data were mainly extracted from reports on international activities in which single or multiple elements of a TBO operation and resulting airspace environment were tested in revenue service using existing technologies and procedures, and/or short-term adoption of more advanced procedures. Other data came from reports of "paper trials" in which "what if" analyses were carried out compared with conventional operations. Available benefits from improved arrival operations were also captured since improvements in oceanic predictability are expected to increase the probability of achieving optimum descent profiles and reduced arrival delay.

The expected benefits associated with each Oceanic TBO Concept area were associated with the phase of flight and/or region in which they were estimated. The expected benefits are summarized in Table 1. Most of the benefit mechanisms are expected to facilitate more fuel and/or cost-efficient routes which, in turn, will allow flight operators to reduce fuel loads, resulting in potentially significant revenue increases. Other key benefits include improved strategic planning and a more efficient 4DT amendment process, resulting in fewer tactical 4DT changes by ATC separate from the 4DT negotiation and amendment process.

Table 1: Summary of benefit mechanisms and expected benefits of the Oceanic TBO concept

\begin{tabular}{|c|c|c|}
\hline Benefit Area & Benefit Mechanism & Benefits \\
\hline $\begin{array}{l}\text { Increased use of } \\
\text { UPRs and 4DTs }\end{array}$ & $\begin{array}{l}\text { Flights use trajectories closer to the } \\
\text { flight operator's preferred } 4 \mathrm{DT}\end{array}$ & $\begin{array}{l}\text { - More fuel/cost efficient routes } \\
\text { - Reduced fuel loads }\end{array}$ \\
\hline $\begin{array}{l}\text { Better support } \\
\text { for } 4 \mathrm{DT} \\
\text { amendments }\end{array}$ & $\begin{array}{l}\text { Increase efficiency of individual } \\
\text { flights' 4DTs as more information } \\
\text { becomes available }\end{array}$ & $\begin{array}{l}\text { - More fuel/cost efficient routes } \\
\text { - } \text { Reduced fuel loads } \\
\text { - More efficient 4DT amendment process } \\
\text { - More 4DT amendments requested and } \\
\text { approved }\end{array}$ \\
\hline $\begin{array}{l}\text { Improved CNS } \\
\text { capabilities }\end{array}$ & $\begin{array}{l}\text { Improved CNS capabilities will support } \\
\text { safe reduction of separation minima }\end{array}$ & $\begin{array}{l}\text { - More fuel/cost efficient routes } \\
\text { - Reduced fuel loads } \\
\text { - Increased capacity/ throughput }\end{array}$ \\
\hline $\begin{array}{l}\text { Improved } \\
\text { information } \\
\text { exchange }\end{array}$ & $\begin{array}{l}\text { Increased ability to exchange } \\
\text { information with flight operators }\end{array}$ & $\begin{array}{l}\text { - More fuel/ cost efficient routes } \\
\text { - Reduced fuel loads } \\
\text { - More predictable 4DTs for flight operators } \\
\text { - Fewer tactical 4DT changes by ATC }\end{array}$ \\
\hline $\begin{array}{l}\text { Precision } \\
\text { scheduling } \\
\text { capabilities }\end{array}$ & $\begin{array}{l}\text { Increased trajectory predictability, } \\
\text { reduced tarmac and en route delays, } \\
\text { reduced fuel burn } \\
\text { Improved ability to plan downstream } \\
\text { operations }\end{array}$ & $\begin{array}{l}\text { - More predictable 4DTs for flight operators } \\
\text { - More fuel/ cost efficient routes } \\
\text { - Reduced fuel loads } \\
\text { - Reduced delays }\end{array}$ \\
\hline $\begin{array}{l}\text { Strategic } \\
\text { planning with } \\
\text { 4DTs }\end{array}$ & $\begin{array}{l}\text { Flights use trajectories close to the } \\
\text { flight operator's preferred 4DT }\end{array}$ & $\begin{array}{l}\text { - More fuel/ cost efficient routes } \\
\text { - Reduced fuel loads }\end{array}$ \\
\hline
\end{tabular}

American Institute of Aeronautics and Astronautics 


\begin{tabular}{|l|l|l|}
\hline Benefit Area & Benefit Mechanism & Benefits \\
\hline & & $\bullet$ Fewer filed flight plans rejected by ATSP \\
& & \\
\hline
\end{tabular}

\section{Conclusions and Future Research Needs}

This small-scale research activity identified current shortfalls and generated questions that should be further investigated to support more efficient trajectories in oceanic (and domestic) airspace and better accommodation of flight operators' preferences. These are briefly discussed here.

\section{A. Strategic Planning Using 4DTs}

Four-dimensional trajectories have been the subject of much research. However, the operational community remains skeptical of their use, chiefly because of the precision expected to be required in a highly dynamic environment. The use of 4DTs for strategic planning has been proposed, ${ }^{5}$ but that proposal provided limited flexibility for coordinating constraints and negotiating trajectories. A more flexible approach would use a collection of proposed 4DTs and characteristics of 4DT uncertainty to derive a probabilistic demand forecast that would support identification of ATM constraints if expected demand will exceed expected capacity. Such constraints can be tuned to balance planning uncertainty with controller complexity without over-constraining traffic flows.

In addition to strategic ATM planning using 4DTs, more research is needed into effective flight planning for TBO when there is significant 4DT uncertainty. For example, stakeholders stressed that the optimal vertical profile is difficult to predict with certainty before departure. There may be a way to improve efficiency even if there is considerable uncertainty in the vertical profile, such as by adopting procedures for flight crews to update the 4DT based on information such as weight and balance and climb-out performance. Such an update would be more effective if the re-optimized 4DT also considered updated winds aloft data, and procedures will still be needed for tactical coordination between flight crews and controllers to account for any remaining trajectory uncertainty.

\section{B. Multi-Party Trajectory Negotiation}

Flight planning for oceanic operations almost always requires some level of international coordination. Oceanic TBO includes a concept for supporting multi-party trajectory negotiation that would allow all affected FIRs to evaluate a proposed trajectory and accept it as is or add constraints that the trajectory must meet (see Figure 2). Such a capability would support FAA as well as ICAO collaborative flight planning and data exchange goals. It would require updated flight and flow data from all affected FIRs, and would need to support coordination with ASPs that had not yet upgraded their data exchange capabilities. It also should decrease the time required to coordinate trajectory amendments and the workload associated with such tasks for flight operator and ASP personnel.

\section{Updating and Amending 4DTs}

The Oceanic TBO concept depends on improved support for updating and amending a 4DT. Such updates may come from the aircraft as downloaded aircraft performance or trajectory intent, from the FOC as a proposed 4DT amendment, or from the ASP as additional (or relaxed) constraints. Such updates are critical for maintaining sufficient information in the system and may lead to trajectory negotiation. Procedures and capabilities for providing these updates should be specified, including situations in which such an update may be required or prohibited.

One such constraint might be a controlled arrival time at a metering fix based on arrival metering operations extended into oceanic airspace. Much of this could be accomplished using current capabilities and updated procedures. These procedures should be defined and evaluated for safety and efficiency.

\section{Tradeoffs Associated with Increased Use of UPRs}

Increased use of UPRs is a key aspect of the Oceanic TBO concept. The benefits of UPRs have been evaluated in previous efforts, ${ }^{15,16}$ but those evaluations have not looked deeply enough at the effects of one flight's UPR on other flights' trajectories and on controller complexity (although Ref. 15 did estimate changes in sector traffic counts). More in-depth analysis is needed of the effect of UPRs in various airspaces. In particular, the complexity of sector traffic flows resulting from UPRs needs to be evaluated to support analysis of the tradeoffs between more flexible routing and controller workload. There may be a saturation point where flows will need to be organized to maintain system efficiency, particularly as separation minima continue to be reduced and equipage and traffic volume increase. Note that there also may be a saturation point for reducing separation minima.

American Institute of Aeronautics and Astronautics 
Together, these initiatives are expected to support the complete adoption of TBO in oceanic airspace, improving trajectory efficiency and allowing flight operators to fly trajectories closer to the optimal 4DT for each aircraft.

\section{Acknowledgments}

This work was performed under contract NNL14AF42P, issued to Mosaic ATM as a result of a competitive NRA solicitation. We are grateful to NASA Langley Research Center for sponsoring this work and to Tom Graff and Ken Jones for their technical review and insights. We thank the Stakeholder Feedback Activity participants who provided their time and operational expertise. We acknowledge the significant contributions of John Brown to the identification of shortfalls, development of the concept, and valuable comments on early drafts of this paper.

\section{References}

1 FAA."NextGen Implementation Plan," Federal Aviation Administration: Washington, DC, 2015.

2 ICAO."Global Air Navigation Plan, 2013-2028," International Civil Aviation Organization: Montreal, 2013.

3 JPDO."Concept of Operations for the Next Generation Air Transportation System, Version 3.2," Joint Planning and Development Office: Washington, DC, 2010.

4 FAA."Order JO7110.65V Air Traffic Control," Department of Transportation: Washington, DC, 2014.

5 Mitchell, T. and Ohsfeldt, M. "Strategic Pre-Departure Coordination with Stochastic Trajectory Modeling," 2012 Integrated Communications Navigation and Surveillance (ICNS) Conference, IEEE, Herndon, VA, 2012.

6 Rebollo, J. and Brinton, C. "Brownian Motion Delay Model for the Integration of Multiple Traffic Management Initiatives," 11th USA/Europe Air Traffic Management Research and Development Seminar (ATM2015), Eurocontrol, Lisbon, 2015.

7 ICAO."Manual on Flight and Flow - Information for a Collaborative Environment (FF-ICE)," ICAO Doc 9965: Montreal, 2012.

8 FAA."Pacific Project Study," FAA Oceanic Integration and Interoperability Facility (OIIF) Lab and FAA Oceanic and Offshore Services, 2012.

9 FAA."Track Advisory User's Guide for Dispatchers," Oakland ARTCC Traffic Management Unit, Anchorage ARTCC Traffic Management Unit: Fremont, CA, 2014f.

10 ICAO."Global Operational Data Link Document (GOLD)," International Civil Aviation Org.: Montreal, 2013.

11 Boeing Company and Air New Zealand."Dynamic Airborne Reroute Procedure (DARP) Automation," International Pacific ATC Coordinating Group: Washington, 2014.

12 McNally, D., Sheth, K., Gong, C., Love, J., Lee, C., Sahlman, S., and Cheng, J.-H. "Dynamic Weather Routes: A Weather Avoidance System For Near-term Trajectory-based Operations," 28th Congress of the International Council of the Aeronautical Sciences, Optimage Ltd., Brisbane, Australia, 2012.

13 Sheth, K., McNally, D., Morando, A., Clymer, A., Lock, J., and Petersen, J. "Benefits Analysis of Multi-Center Dynamic Weather Reroutes," 14th AIAA Aviation Technology, Integration, and Operations Conference, AIAA, Atlanta, GA, 2014.

14 Wing, D. J. "Achieving TASAR Operational Readiness," 15th AIAA Aviation Technology, Integration, and Operations Conference, AIAA, Dallas, 2015.

15 Grabbe, S., Sridhar, B., and Cheng, N. "Central East Pacific Flight Routing," AIAA Guidance, Navigation, and Control Conference, AIAA, Keystone, CO, 2006.

16 Sridhar, B., Chen, N. Y., Rodionova, O., Delahaye, D., Ng, H. K., and Linke, F. "Strategic Planning of Efficient Oceanic Flights," Eleventh USA/Europe Air Traffic Management Research and Development Seminar (ATM2015), Eurocontrol, Lisbon, 2015.

17 ASPIRE."2014 Annual Report," 2014.

18 FAA."Oceanic Work Group Meeting Synopsis, June 18, 2014," 2014.

19 Liu, S."Atlantic Interoperability Initiative to Reduce Emissions (AIRE) - Environmental Program Status," 2009.

20 FAA. "User Preferred Route (UPR) and PACOTS Update," 27th Meeting of the Informal South Pacific ATS Coordinating Group (ISPACG/27), Auckland, NZ, 2013.

American Institute of Aeronautics and Astronautics 
21 Pacific Project. "Pacific Project (PPT/7) Meeting Report," 18th Meeting of the Cross Polar Trans-East ATM Providers Working Group (CPWG/18), Paris, 2014.

22 JPDO."Trajectory-Based Operations (TBO) Study Team Report," Next Generation Air Transportation System Joint Planning and Development Office: Washington, DC, 2011. 